\title{
Reflection of Electromagnetic Waves From Thin Ionized Gaseous Layers
}

\author{
F. H. Northover \\ Contribution from Department of Mathematics, Carleton University, Ottawa, Canada
}

(Received March 24, 1961; revised August 3, 1961)

\begin{abstract}
The reflection properties of thin ionized layers are examined, and earlier work on the parabolic layer, which seems unsatisfactory in some respects, is placed upon a more rigorous footing. Where the layer extends over most of the transmission path, an approximate estimate is made of the rate of attentuation of the waves around the curved earth. In this way it seems possible to account for the freak long distance transmission of very high frequency radio wave which is sometimes observed: a particular example of this is considered in detail.
\end{abstract}

\section{Introduction}

It is the usual practice in ionospheric analysis to make use of optical methods of treatment. While this technique is satisfactory for ionospheric layers whose thicknesses are great compared with the wavelength of the critical penetration frequency of the layer, results thus obtained are likely to be misleading when this condition does not apply.

It is now experimentally established that thin $E$ and sporadic $-E$ layers are capable of causing long distance propagation of $\mathrm{HF}$ radio waves through reflections at oblique incidence. One of the first to investigate these reflections theoretically was Hartree [1929], who considered a layer distribution law in which the electron density increased linearly and symmetrically from the two edges to a peak at the middle. A simpler formula for the reflection coefficient for this case was given in an independent investigation by Beghian and Northover [1943]. ${ }^{1}$ In 1930 other distribution laws were examined by Epstein [1930] and, later on, the parabolic law was investigated by Rydbeck [1943]. Although the results of these last two writers explain the main features of the reflection phenomena for thick layers, their analysis becomes completely erroneous for thin layers. The error arises through their attempts to short circuit the four equations expressing the two boundary conditions at each layer edge by attempting to force a physical interpretation upon what after all amounts to nothing more nor less than a theorem of pure mathematics. The consequent erroneous conclusions show up much more clearly in the Rydbeck paper because this is concerned with only one specific case - the parabolic distribution. The formula (9) on page 347 of his paper for the reflection coefficient $R$ gives the absurd result that $R \rightarrow 1 / \sqrt{2}$ as the layer thickness tends to zero. See further, appendix 1 .

On simple ray theory, reflection from the underside of a sporadic - E' layer (thin ionized layer

\footnotetext{
1 See end of section 3
}

occurring sporadically) would be almost complete for frequencies below the maximum usable frequency (MUF) but almost negligible for higher frequencies. Vertical incidence measurements, however, have shown that a certain amount of reflection often occurs at frequencies bigher than the MUF and, for this reason, it may be difficult to decide the exact value of the critical frequency of the layer. As far as is known to the writer, few systematic observations have been made at oblique incidence, but it is evident both from theory and practical experience that this phenomenon may become increasingly important for rays incident at high obliquities upon the layer. In particular, serious modifications to the skip distances calculated on ray theory may occur when the layer is thin.

\section{Reflection of Plane Waves}

\subsection{Preliminary Analysis}

If the layer is horizontally stratified, and the waves horizontally polarized, the components of the electric vector satisfy the two-dimensional wave equation

$$
\frac{\partial^{2} X}{\partial y^{2}}+\frac{\partial^{2} X}{\partial z^{2}}+h^{2} X=0
$$

where $\partial y$ is horizontal, $\partial z$ is vertical, the usual exponential time factor is assumed, i.e., $\exp (-i \omega t)$, and

$$
h^{2}=\frac{4 \pi^{2} f^{2}}{C^{2}}\left(1-\frac{f_{N}^{2}}{f^{2}+i \nu f}\right)
$$

$f_{N}$ being the plasma frequency at height $z$ and $\nu$ being the collision frequency. Here $f$ is the wave frequency and $c$ the velocity of light and we are neglecting the effects of the earth's magnetic field.

In the sporadic $-E$ region $\nu$ can usually be neglected in comparison with $f$ for $f$ much greater than a megacycle. Hence, neglecting $\nu / f$, and writing

$$
X=X_{0} e^{\frac{2 \pi i f}{c} y \sin \theta} F(z)
$$


so that $\theta$ is the angle which the incident wave makes with the normal, we have,

$$
\frac{d^{2} F}{d z^{2}}+\frac{4 \pi^{2} f^{2}}{c^{2}}\left(\cos ^{2} \theta-\frac{f_{N}^{2}}{f^{2}}\right) F=0
$$

The only type of ionic density distribution which is amenable 2 to rigorous mathematical analysis is the parabolic law investigated by Rydbeck in 1943, which may sometimes represent an approximation to the truth. Taking the origin at the center of the layer, this may be expressed as

$$
N=N_{o}\left(1-\frac{z^{2}}{\tau^{2}}\right)
$$

where $2 \tau$ is the thickness of the layer, $N$ is the ionic density at any point within it, and $N_{o}$ is the maximum value of $N$ within the layer.

It can be shown that the plasma frequency $f_{N}$ is given by

$$
f_{N}^{2}=N e^{2} / \pi m
$$

where $e$ is the charge on the ion and $m$ is its mass: hence,

$$
f_{N}^{2}=f_{N_{m}}^{2}\left(1-\frac{z^{2}}{\tau^{2}}\right)
$$

where $f_{N_{m}}$ is the maximum value of $f_{N}$.

By (6) and (3) the equation satisfied by $F(z)$ within the layer is

$$
\frac{d^{2} F}{d \gamma^{2}}+\mu_{N_{m}}\left\{\left(\Lambda^{2}-1\right)+\gamma^{2}\right\} F=0
$$

where

$$
\Lambda=\frac{f}{f_{N_{m}} \sec \theta} ; \quad \mu_{N_{m}}=\frac{2 \pi \tau}{\lambda_{N_{m}}} ; \quad \gamma=\frac{z}{\tau} .
$$

\subsection{Physical Meaning of $\Lambda$}

It can be shown from (7) that. if the thickness of the layer is large compared with the wavelength of the radio frequency $f$ an upward traveling wave near the center of the layer has the approximate form

$$
\exp \frac{2 \pi i f}{c}\left\{y \sin \theta+z \cos \theta\left(1-1 / \Lambda^{2}\right)^{1 / 2}\right\} \text {. }
$$

Accordingly, if $\Lambda<\mid$ this wave will be strongly attenuated which means that strong reflection has occured lower down. Hence $f_{N_{m}} \sec \theta$ is the oblique penetration frequency when the layer thickness is very large compared with the wavelength of the frequency used, so that $\Lambda$ denotes the ratio of the frequency used to the maximum usable frequency for thick layers appropriate to the particular obliquity of incidence under consideration.

When the thickness of the layer is of the order of $\lambda_{N_{m}}$ however, it is found that the transition from 2 Because the parabolic cylinder functions are relatively well known, c.f.
Whittaker and Watson. reflection to transmission as $\Lambda$ increases through unity is a good deal more gradual and, further, that if $2 \tau$ is much less than $0.2 \lambda_{N_{m}}$, reflection is slight for all values of $\Lambda$.

\subsection{Calculation of the Reflection Coefficent}

Let the wave incident upon the layer be represented by $\exp \left\{\frac{2 \pi f}{c} z \cos \theta\right\}$. If $R, \bar{R}$ are the complex reflection and transmission coefficients respectively, then the reflected and transmitted waves are represented by

$$
R e^{-\frac{2 \pi i f}{c} z \cos \theta}, \quad \bar{R} e^{\frac{2 \pi i f}{c} z \cos \theta}
$$

respectively.

If $u(\gamma), v(\gamma)$ denote any pair of fundamental solutions of (7), the boundary conditions at the layer give

$$
\left.\begin{array}{rl}
e^{\frac{i \omega}{c} r \cos \theta}+R e^{\frac{i \omega}{c} \tau \cos \theta} & =A u(-1)+B v(-1) \\
\bar{R} e^{\frac{i \omega}{c} \tau \cos \theta} & =A u(1)+B v(1) \\
\frac{i \tau \omega}{c} \cos \theta\left(e^{-\frac{i \omega}{c} \tau \cos \theta}-R e^{\frac{i \omega}{c} \tau \cos \theta}\right) & =A u^{\prime}(-1)+B v^{\prime}(-1) \\
\frac{i \tau \omega}{c} \cos \theta \bar{R} e^{\frac{i \omega}{c} \tau \cos \theta} & =A u^{\prime}(1)+B v^{\prime}(1) .
\end{array}\right\}
$$

where

$$
\omega=2 \pi f \text {. }
$$

Writing

$$
R_{1}=R e^{\frac{2 i \omega}{c} \tau \cos \theta}
$$

and

$$
\begin{gathered}
L=u(1) v(-1)-u(-1) v(1) \\
\mu M_{1}=u^{\prime}(1) v(-1)-u(-1) v^{\prime}(1) \\
\mu M_{2}=u(1) v^{\prime}(-1)-u^{\prime}(-1) v(1) \\
\mu^{2} N=u^{\prime}(1) v^{\prime}(-1)-u^{\prime}(-1) v^{\prime}(1)
\end{gathered}
$$

where

$$
\mu=2 \pi \tau / \lambda
$$

we have

$$
R_{1}=\frac{\left(L \cos ^{2} \theta-N\right)+i\left(M_{1}+M_{2}\right) \cos \theta}{\left(L \cos ^{2} \theta+N\right)+i\left(M_{1}-M_{2}\right) \cos \theta}
$$

In the most important cases physically the solutions of (7) can be approximated to by the Jeffrey's (W.K.B.) method of asymptotic approximation. This method was afterwards extended by R. E. Langer [1937]. It consists of obtaining an asymptotic solution of the standard equation

in the form

$$
\Omega^{\prime \prime}+\varphi \Omega=0
$$

$$
\varphi^{-1 / 4} e^{ \pm i \int^{\Gamma} \sqrt{\phi} d \gamma^{\prime}}
$$


It can be shown that (15) is useful only when both $\left|5 \varphi^{\prime 2} / 32 \varphi^{3}\right|$ and $\left|\varphi^{\prime \prime} / 4 \varphi^{2}\right|$ are small, ${ }^{3}$ and that a further refinement of $(15)$ is

$\varphi^{-1 / 4} \exp \left[ \pm i \int^{\gamma}\left\{\sqrt{\varphi}-\varphi^{\prime \prime} / 8 \varphi^{3 / 2}+5 \varphi^{\prime 2} / 32 \varphi^{5 / 2}\right\} d \gamma\right]$

We require only the approximate values of $u$ and $v$ near the edges of the layer $(\gamma= \pm 1)$ and then the above conditions of validity for (15) require that

and

$$
\begin{array}{ll}
\mu_{N_{m}} \Lambda^{2}>2 & \text { if } \Lambda>1 \\
\mu_{N_{m}} \Lambda^{3}>2 & \text { if } \Lambda<1
\end{array}
$$

\subsection{Calculation of $u(\gamma)$ and $v(\gamma)$ at the Layer Edges}

The approximate formulas for $u$ and $v$ near $\gamma=+1$ cannot be used near $\gamma=-1$ on account of a Stokes phenomenon in the asymptotic solution (15) as we pass through the layer. We shall obtain the required continuation of $u(\gamma)$ and $v(\gamma)$ for negative values of $\gamma$ from the theory of the Weber Parabolic Cylinder Function [Whittaker and Watson, 1927].

Equation (7) can be written

$$
\frac{d^{2} F}{d v^{2}}+\left(\alpha+\frac{v^{2}}{4}\right) F=0
$$

where

$$
v=\gamma\left(2 \mu_{N_{m}}\right)^{1 / 2} ; \quad \alpha=\frac{1}{2} \mu_{N_{m}}\left(\Lambda^{2}-1\right)
$$

and solutions are

$$
D_{ \pm i \alpha-\frac{1}{2}}\left(v e^{\mp \frac{i \pi}{4}}\right) \text { and } D_{ \pm i \alpha-\frac{1}{2}}\left(-v e^{\mp \frac{i \pi}{4}}\right) .
$$

We shall take, for $u(\gamma)$ and $v(\gamma)$, the conjugate functions

$$
\begin{aligned}
& u(\gamma)=\sqrt{2} e^{-\frac{\pi \alpha}{4}-\frac{i \pi}{8}} D_{i \alpha-\frac{1}{2}}\left\{\gamma\left(2 \mu_{N_{m}}\right)^{\frac{1}{2}} e^{-\frac{i \pi}{4}}\right\} \\
& v(\gamma)=\overline{u(\gamma)} \quad(\gamma \text { real }) .
\end{aligned}
$$

The Jeffrey's approximations to (18) are found to be

$$
\frac{\left\{\frac{v}{2}+\left(\alpha+\frac{v^{2}}{4}\right)^{\frac{1}{2}}\right\}^{ \pm i \alpha}}{\left(\alpha+\frac{v^{2}}{4}\right)^{\frac{1}{4}}} e^{ \pm \frac{i v}{2}\left(\alpha+\frac{v^{2}}{4}\right)^{\frac{1}{2}}}
$$

Now if $v \rightarrow \infty$ and $\alpha$ is fixed, the well known formulas

$$
D_{n}(z) \sim e^{-\frac{1}{2} z^{2}} z^{n}\left\{1+0\left(n^{2} / z^{2}\right)\right\}
$$

valid when $z$ is large, $n$ fixed and $|\arg z|<3 \pi / 4$ gives

$$
u(\gamma) \sim \sqrt{2} v^{i \alpha-\frac{1}{2}} e^{\frac{i v^{2}}{4}}
$$

But no Stokes line is crossed between $\gamma=+1$ and $\gamma=+\infty$, since $\alpha+\frac{v^{2}}{4}$ remains positive. Hence, by (20 and (22) we have

$$
u(\gamma) \sim \frac{\left\{\frac{v}{2}+\left(\alpha+\frac{v^{2}}{4}\right)^{\frac{1}{2}}\right\}^{i \alpha}}{\left(\alpha+\frac{v^{2}}{4}\right)^{\frac{1}{4}}} e^{\frac{i v}{2}\left(\alpha+\frac{v^{2}}{4}\right)^{\frac{1}{2}}} .
$$

This formula is valid near the upper edge of the layer $(\gamma=1)$ subject to the conditions (17). Now

$$
\begin{aligned}
D_{i \alpha-\frac{1}{2}}\left(v e^{\frac{3 i \pi}{4}}\right)= & e^{\left(i \alpha-\frac{1}{2}\right) i \pi} \cdot D_{i \alpha-\frac{1}{2}}\left(v e^{\left.-\frac{i \pi}{4}\right)}\right. \\
& +\frac{\sqrt{2 \pi}}{\Gamma\left(-i \alpha+\frac{1}{2}\right)} e^{\frac{1}{2}\left(i \alpha+\frac{1}{2}\right) i \pi} D_{-i \alpha-\frac{1}{2}}\left(v e^{\frac{i \pi}{4}}\right) .
\end{aligned}
$$

Hence for $\quad-\frac{1}{2} \pi<\arg v<\pi$,

$u(-\gamma)=e^{\frac{5 i \pi}{4}\left(i \alpha-\frac{1}{2}\right)} D_{i \alpha-\frac{1}{2}}\left(v e^{-\frac{i \pi}{4}}\right)$

$$
\begin{array}{r}
+\frac{\sqrt{2 \pi}}{\Gamma\left(-i \alpha+\frac{1}{2}\right)} e^{\frac{3 i \pi}{4}\left(i \alpha+\frac{1}{2}\right)} D_{-i \alpha-\frac{1}{2}}\left(v e^{\frac{i \pi}{4}}\right) \\
=e^{i \pi\left(i \alpha-\frac{1}{2}\right)} u(\gamma)+\frac{\sqrt{2 \pi}}{\Gamma\left(-i \alpha+\frac{1}{2}\right)} e^{\frac{1}{2}\left(i \alpha+\frac{1}{2}\right) i \pi} v(\gamma) \\
\therefore u^{\prime}(-\gamma)=i e^{-\pi \alpha}\left\{u^{\prime}(\gamma)+\frac{\sqrt{2 \pi}}{\Gamma\left(-i \alpha+\frac{1}{2}\right)} e^{\frac{\pi \alpha}{2}+\frac{3 i \pi}{4}} v^{\prime}(\gamma)\right\} .
\end{array}
$$

Again, from (23)

$$
u^{\prime}(\gamma) \sim i\left(2 \mu_{N_{m}}\right)^{\frac{1}{2}}\left(\alpha+\frac{v^{2}}{4}\right)^{\frac{1}{4}}\left\{\frac{v}{2}+\left(\alpha+\frac{v^{2}}{4}\right)^{\frac{1}{2}}\right\}^{i \alpha} e^{\frac{i v}{2}\left(\alpha+\frac{v^{2}}{4}\right)^{\frac{1}{2}}}
$$

the error being of the order of the error incurred in using Jeffery's approximations. Hence, (25) and (26) give, neglecting error terms of order

$$
\begin{gathered}
\left(2 \mu_{N_{m}} \Lambda^{3}\right)^{-1} \\
u(1)=\frac{(\Lambda+1)^{i \alpha}}{\sqrt{\Lambda}}\left(\frac{\mu_{N_{m}}}{2}\right)^{\frac{1}{2} i \alpha-\frac{1}{4}} e^{\frac{1}{2} i \mu_{N_{m}} \Lambda} \\
u(-1)=-i e^{-\pi \alpha} \frac{(\Lambda+1)^{i \alpha}}{\sqrt{\Lambda}}\left(\frac{\mu_{N_{m}}}{2}\right)^{\frac{1}{2} i \alpha-\frac{1}{2}} e^{\frac{1}{2} \mu_{N_{m}}{ }^{i \Lambda}} \\
+\frac{\sqrt{2 \pi}}{\Gamma\left(-i \alpha+{ }^{1} / 2\right)} e^{\frac{-\pi \alpha}{2}+\frac{i \pi}{4} \cdot \frac{(\Lambda+1)^{-i \alpha}}{\sqrt{\Lambda}}} \\
\left(\frac{\mu_{N_{m}}}{2}\right)^{-\frac{1}{2} i \alpha-\frac{1}{6}} e^{-\frac{1}{2} i \mu_{N m} \Lambda}
\end{gathered}
$$




$$
\begin{gathered}
u^{\prime}(1)=i(\Lambda+1)^{i \alpha}\left(\frac{\mu_{N_{m}}}{2}\right)^{\frac{1}{2} i \alpha+\frac{1}{4}} \sqrt{\Lambda} e^{\frac{1}{2} \mu_{N_{m}}}{ }^{\Lambda} \cdot \sqrt{2 \mu_{N_{m}}} \\
u^{\prime}(-1)=i\left\{i e^{-\pi \alpha}(\Lambda+1)^{i \alpha}\left(\frac{\mu_{N_{m}}}{2}\right)^{\frac{i \alpha}{2}+\frac{1}{4}} \sqrt{\Lambda} \cdot e^{\frac{1}{2} \mu_{N_{m}} \Lambda}\right. \\
+\frac{\sqrt{2 \pi}}{\Gamma\left(-i \alpha+\frac{1}{2}\right)} e^{-\frac{\pi \alpha}{2}+\frac{i \pi}{4}}(\Lambda+1)^{-i \alpha}\left(\frac{\mu_{N_{m}}}{2}\right)^{-\frac{i \alpha}{2}-\frac{1}{4}} \\
\left.\cdot \sqrt{\Lambda} e^{-\frac{1}{2} i \mu N_{m} \Lambda}\right\} \times \sqrt{2 \mu_{N_{m}}} .
\end{gathered}
$$

Hence

$$
\begin{aligned}
L=u(1) \bar{u}(-1)-\bar{u}(1) u(-1) \\
=\frac{1}{\Lambda}\left(\frac{\mu_{N_{m}}}{2}\right)^{-1 / 2} e^{-\pi \alpha} \\
\quad\left\{i+\frac{\sqrt{2 \pi}}{\Gamma\left(i \alpha+\frac{1}{2}\right)} e^{\frac{\pi \alpha}{2}-\frac{i \pi}{4}}(\Lambda+1)^{2 i \alpha}\left(\frac{\mu_{N_{m}}}{2}\right)^{i \alpha} e^{i \mu_{N_{m}} \Lambda}\right\}
\end{aligned}
$$

- the conjugate function

$=\frac{1}{\Lambda}\left(\frac{\mu_{N m}}{2}\right)^{-1 / 2} e^{-\pi_{\alpha}}(2 i+2 i a \sin \varphi)$

where

$$
a e^{i \varphi}=\frac{\sqrt{2 \pi}}{\Gamma\left(i \alpha+\frac{1}{2}\right)} e^{\frac{\pi \alpha}{2}-\frac{i \pi}{2}}(\Lambda+1)^{2 i \alpha}\left(\frac{\mu_{N_{m}}}{2}\right)^{i \alpha} e^{i \mu_{N_{m}}{ }^{\Lambda}} .
$$

Similarly, we find

$$
\begin{gathered}
\mu M_{1}=\sqrt{2 \mu_{c_{m}}} \cdot 2 i a e^{-\pi_{\alpha}} \cos \varphi=-\mu M_{2} \\
\mu^{2} N=\Lambda\left(\frac{\mu_{N_{m}}}{2}\right)^{1 / 2} 2 \mu_{N_{m}} e^{-\pi_{\alpha}}(-2 i+2 a i \sin \varphi)
\end{gathered}
$$

Now

$$
\left.\begin{array}{c}
\mu^{-1} \Lambda \mu_{N_{m}}=\cos \theta ; \text { hence } \\
\omega L=1+a \sin \varphi \\
\omega M_{1}=a \cos \theta \cos \varphi=-\omega M_{2} \\
\omega N=\cos ^{2} \theta(a \sin \varphi-1)
\end{array}\right\}
$$

where $\omega$ is a constant factor $_{3}$

This gives

$$
\begin{gathered}
R_{1}=\frac{1}{a i} e^{i \phi} \\
\therefore\left|R_{1}\right|=1 / a=\left(1+e^{2 \pi \alpha}\right)^{-1 / 2} .
\end{gathered}
$$

This formula is true subject to the conditions (17), so long as $1 / a$ is large compared with the error incurred in using Jeffrey's approximations. Using the refinement (16) it is possible to show that

$$
|R|=1 / a-\left\{1 /\left(2 \mu_{N_{m}} \Lambda^{3}\right)\right\} \cos \varphi+\sigma(\varphi) /\left(4 \mu_{N_{m}}^{2} \Lambda^{4}\right)
$$

where

$$
\sigma(\varphi)<1
$$

Hence, even when $\mu_{N_{m}}$ is not small, there is a wide divergence from $\mathbf{R}$ ydbeck's result ${ }^{4}$ when $\alpha$ exceeds about 0.5 , and the reflection coefficient actually vanishes for values of $\Lambda$ and $\mu_{N_{m}}$ which make $\varphi$ near to an odd multiple of $\frac{1}{2} \pi$.

\subsection{Case when $\mu_{N_{m}}$ is Small-the Series Solutions to (7)}

Equation (7) is

where

$$
\frac{d^{2} \Omega}{d \gamma^{2}}+\left(A+B \gamma^{2}\right) \Omega=0
$$

$$
A=\mu_{N_{m}}^{2}\left(\Lambda^{2}-1\right), \quad B=\mu_{N_{m}}^{2}
$$

and it can be shown that, as far as squares and products of the small quantities $A$ and $B$, two fundamental solutions ${ }^{5}$ of this equation are

$$
\begin{aligned}
u(\gamma) \doteqdot 1-\frac{1}{2} A \gamma^{2} & -\frac{1}{12} B \gamma^{4}+\frac{1}{24} A^{2} \gamma^{4} \\
& +\frac{7}{360} A B \gamma^{6}+\frac{1}{672} B^{2} \gamma^{8} \\
v(\gamma) \doteqdot \gamma-\frac{1}{6} A \gamma^{3}-\frac{1}{20} B \gamma^{5} & +\frac{1}{120} A^{2} \gamma^{5} \\
& +\frac{13}{2520} A B \gamma^{7}+\frac{1}{1440} B^{2} \gamma^{9}
\end{aligned}
$$

Using these approximations to evaluate approximately the functions $L, M_{1}, M_{2}$, and $N$ we find

$$
\begin{aligned}
L & =-2\left\{1-\frac{1}{2} \mu_{N_{m}}^{2}\left(\frac{4}{3} \Lambda^{2}-\frac{16}{15}\right)\right\} \\
M_{1} & =-\frac{1}{\mu_{N_{m}} \Lambda} \cos \theta\left\{1-2 \mu_{N_{m}}^{2}\left(\Lambda^{2}-\frac{2}{3}\right)\right. \\
& \left.\quad+\frac{1}{4} \mu_{N_{m}}^{4}\left(\frac{5}{3} \Lambda^{4}-\frac{101}{45} \Lambda^{2}+\frac{131}{180}\right)\right\} \\
= & -M_{2} \quad \\
N & =-2 \cos ^{2} \theta\left(1-\frac{2}{3 \Lambda^{2}}\right)\left\{1-\frac{1}{2} \mu_{N_{m}}^{2}\left(\Lambda^{2}-\frac{1}{2}\right)\right\}
\end{aligned}
$$

whence

$$
|R|^{2}=\frac{\left\{\frac{2}{3 \Lambda^{2}}-\frac{1}{2} \mu_{N_{m}}^{2}\left(\frac{\Lambda^{3}}{3}+\frac{1}{10}-\frac{1}{3 \Lambda^{2}}\right)\right\}^{2}}{\left\{2-\frac{2}{3 \Lambda^{2}}-\frac{1}{2} \mu_{N_{m}}^{2}\left(\frac{7 \Lambda^{2}}{3}-\frac{67}{30}+\frac{1}{3 \Lambda^{2}}\right)\right\}^{2}+\frac{1}{\mu_{N_{m}}^{2}} \Lambda^{2}\left\{1-2 \mu_{N_{m}}^{2}\left(\Lambda^{2}-\frac{2}{3}\right)+\frac{\mu_{N_{m}}^{4}}{720}\left(307 \Lambda^{4}-404 \Lambda^{2}+131\right)\right\}^{2}}
$$


provided that $\mu_{N_{m}}$ and $2 \mu_{N_{m}}\left(\Lambda^{2}-1\right)$ are both less than unity. The reflection coefficient therefore becomes small if $\mu_{N_{m}} \Gamma<<1$ and tends to unity if $\mu_{N_{m}} / \Gamma>>1$,

\section{Discussion of Results}

The behavior of $|R|$ for the most interesting values of $\mu_{N_{m}}$ and $\Lambda$ is exhibited in the graph. Now, as $\mu_{N_{m}} \rightarrow 0,|R| \rightarrow 0$, and, as $\mu_{N_{m}} \rightarrow \infty$,

$$
\begin{aligned}
& |R| \rightarrow 1 \text { if } \Lambda<1 \\
& \rightarrow 1 / \sqrt{2} \text { if } \Lambda=1 \\
& \rightarrow 0 \text { if } \Lambda>1 .
\end{aligned}
$$

Since $|R|$ is continuous it must, for every fixed $\Lambda>1$, have a maximum value $|R|_{M}$ for some value $\mu_{M}(\Lambda)$, say, of $\mu_{\mathrm{N}_{m}}$ and it can be shown that $|R|_{M}$ and $\mu_{M}$ are steadily decreasing functions of $\Lambda$ when $\Lambda>1$. If we say that $|R|>1 / 3$ for appreciable reflection, we find that the greatest frequency for which good reflection can occur $\left(|R|_{M}>^{1 / 3}\right)$ is given by $\Lambda=1.23$, approximately, the corresponding value of $\mu_{M}$ then being about 1.33. Taking as typical the values $f_{N_{m}}=3 \mathrm{Mc} / \mathrm{s}, h=80 \mathrm{~km}$, we find that the greatest frequency which can suffer appreciable reflection (under the above mentioned criterion) is $23 \mathrm{Mc} / \mathrm{s},{ }^{6}$ provided that the layer thickness is then near $42 \mathrm{~m}$.

When the frequency is near the ray theory MUF (in this case about $19 \mathrm{Mc} / \mathrm{s}$ ), $\Lambda=1$, and $|R|$ exceeds $1 / 3$ only for layer thicknesses greater than $0.16 \lambda_{N_{m}}$. Thus, for $f=3 \mathrm{Mc} / \mathrm{s}$, good reflection can be obtained at the ray theory MUF for layers down to about $16 \mathrm{~m}$ thick.

\subsection{Intense Sporadic-E Formation-Bennington's Figures}

In the case of the intense sporadic $-E$ ionization described by Bennington [1952], $f_{N_{m}}$ may be expected to be as large as $7 \mathrm{Mc} / \mathrm{s}$ for roughly 8 percent of the time during the summer months (c.f., fig. 4 of his paper). It would seem, therefore, to be not unreasonable to take $7 \mathrm{Mc} / \mathrm{s}$ as a typical value for $f_{N_{m}}$ for intense sporadic - $E$ ionization. As the height of this ionization was about $115 \mathrm{~km}$, we now find that the greatest frequency which can suffer appreciable reflection, according to the criteria and theory of the preceding paragraph, is $45 \mathrm{Mc} / \mathrm{s}$, provided that the layer thickness is then near $18 \mathrm{~m}$.

When the frequency is near the ray theory MUF (in this case about $37 \mathrm{Mc} / \mathrm{s}$ ), $\Lambda \doteqdot 1$, and $|R|$ exceeds $1 / 3$ for layer thicknesses greater than $0.16 \lambda N_{m}$. Thus for $f_{N_{m}}=7 \mathrm{Mc} / \mathrm{s}$ good reflection can be obtained at the ray theory MUF for layers down to about $7 \mathrm{~m}$ thick.

This corresponds to the ray which strikes the layer at the greatest possible obliquity and is determined by elementary geometry of the curved earth.

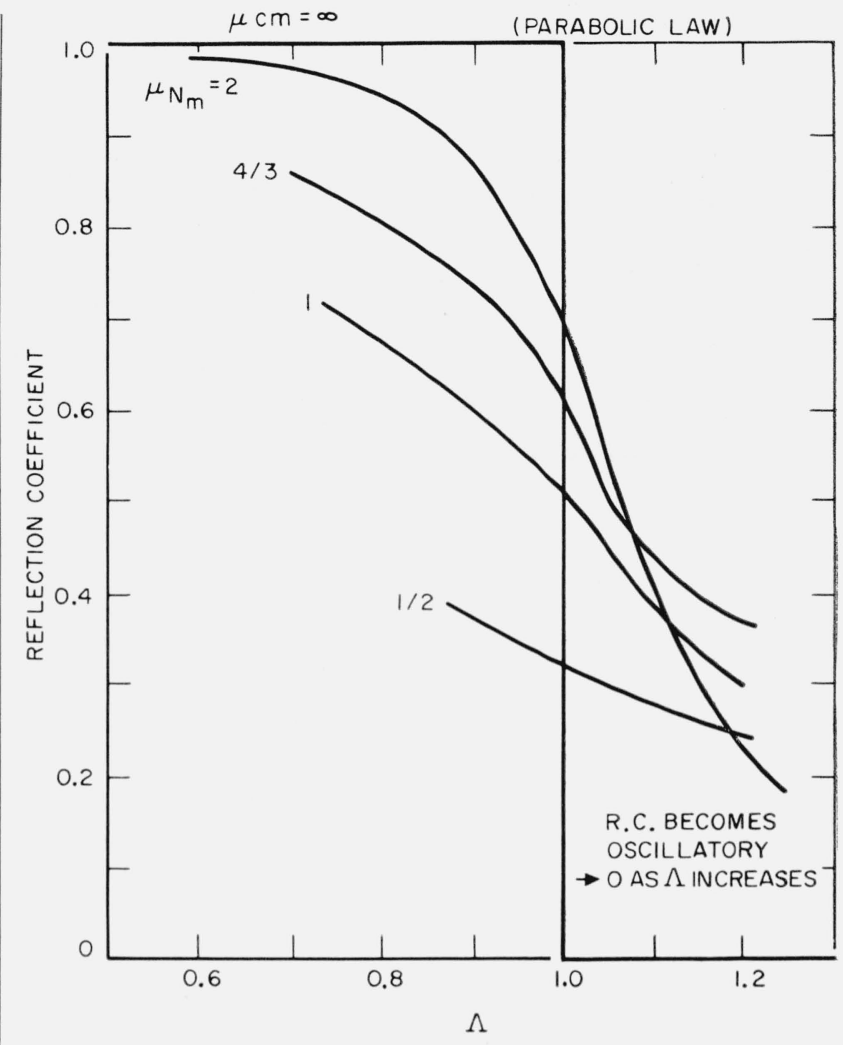

Figure 1. Reflection coefficient versus $\Lambda$.

\subsection{Note on the Linear Law of Ionic Density Distri- bution}

The writer has investigated the linear law of distribution

$$
N=N_{0}\left(1-\frac{|z|}{\tau}\right)
$$

However, as only straightforward mathematical methods are required for what is in this case a straightforward boundary value problem we shall only quote below the result obtained for the reflection coefficient of such a layer. It is

where

$$
|R|=\frac{1}{\sqrt{1+\chi^{2}}}
$$

where

$$
\chi=\frac{27}{4 \pi^{2} \mu_{N_{m}}^{2} \Lambda^{3}\left(\Lambda^{2}-1\right)^{3 / 2} k}
$$

$$
\begin{aligned}
k=\left(J_{1 / 3} J_{-1 / 3}^{*}-J_{1 / 3}^{*} J_{-1 / 3}\right)\left(J_{1 / 3} J_{2 / 3}^{*}+J_{-1 / 3} J_{-2 / 3}^{*}\right) \\
+\left(J_{2 / 3}^{*} J_{-2 / 3}-J_{-2 / 3}^{*} J_{2 / 3}\right)\left(J_{1 / 3}^{*} J_{2 / 3}+J_{-1 / 3}^{*} J_{-2 / 3}\right)
\end{aligned}
$$

$J_{n}$ is written for $J_{n}\left(\frac{2}{3} \mu_{N_{m}} \Lambda^{3}\right)$

$J_{n}^{*}$ is written for $J_{n}\left\{\frac{2}{3} \mu_{N_{m}}\left(\Lambda^{2}-1\right)^{3 / 2}\right\}$. 


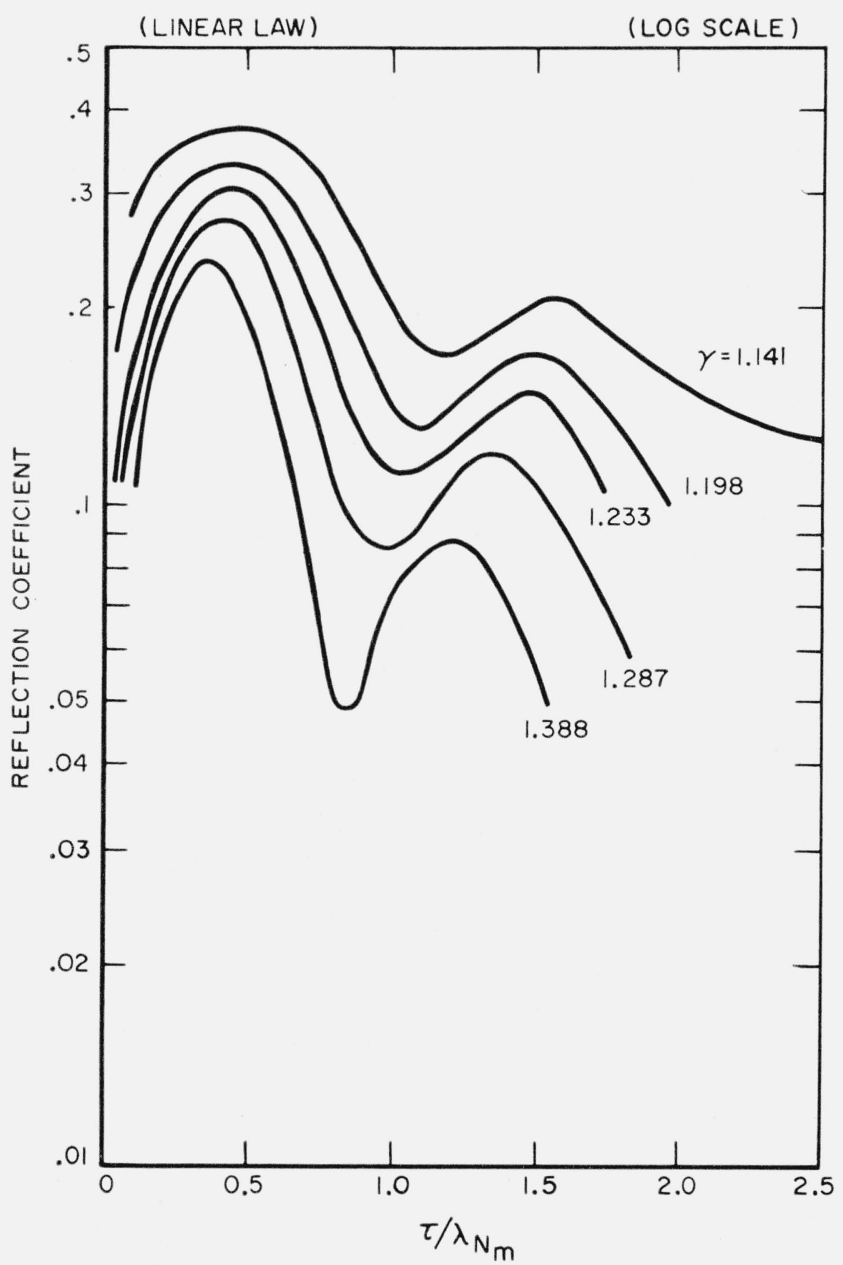

Figure 2. Reflection coefficient versus $\tau / \lambda_{N m}$.

\section{Attenuation of the Waves Around a Curved Earth}

In most cases of propagation by reflection from sporadic-E layers the ionization is confined to the locality above the mid-point of the path. Since, however, sporadic - $E$ formation tends to be most marked near noon each day (c.f., Bennington's paper. fig. 2), it might be possible for sporadic- $E$ ionization to develop over much of the transmission path if this lay nearly along a meridian. If the sporadic-E was well developed over the widely separated points necessary for multi-hop transmission (see appendix 2), the field strength over the transmission path would be approximately the same as that which would be due to a thin ionized layer concentric with the earth. We therefore proceed to investigate the latter problem and in this way attempt to account for the extraordinary reception of the BBC's 1949 Oxford-Cambridge Boat Race television transmission in Capetown, and other more recent similar freak transmissions. ${ }^{7}$

\subsection{Field Beneath a Parabolically Ionized Layer}

Let the field be due to a horizontal dipole situated below the laver at height $H$ above the ground, and be emitting waves of frequency $f$. The effective dielectric constant below and above the layer will be taken as unity, and, within the layer, as approximately $1=f_{N}^{2} / f^{2}$ where $f_{N}^{2}=f_{N m}^{2}\left(1-\gamma^{2}\right)$ see (1a) and $(6)\}$.

We take spherical polar coordinates $(r, \theta, \phi)$ with origin at the earth's center, initial line $(\theta=0)$ along the line joining the earth's center to the transmitter, and the direction of the dipole axis parallel to $\phi=\left.\pi\right|_{2}$. It can then be shown [Northover, 1952] that the tangential electric field $E_{\phi}$ over a perfectly conducting earth (this assumption is a good approximation in most cases of $\mathrm{HF}$ propagation), is,

$$
\frac{8 \pi^{2} H z}{R \lambda^{3 / 2}}\left(\frac{3 \Gamma_{1}}{C d}\right)^{\frac{1}{3}} \sum_{1}^{\infty} \mathscr{F}_{j}^{-1} \exp \left\{-\left(X_{j} i+Y_{j}\right) x^{\frac{1}{2} \theta}\right\}
$$

where $H, z$ are the transmitter and receiver heights, $R$ is the earth's radius, $\lambda$ the wavelength, $C$ the velocity of light, $d=R \theta, \Gamma$, the radiated power, and $X_{j}, Y_{j}$ are numbers given by the equation

where

$$
\zeta_{\nu-1 / 2}(x) / \eta_{\nu-1 / 2}(x)=k
$$

$$
\nu_{j}=x\left(1+\epsilon_{j}\right), \quad x=2 \pi R / \lambda, \quad x^{2 / 3} \epsilon_{j}=x_{j}-i Y_{j} .
$$

Here

$$
k=\frac{N\left\{\zeta_{\nu-1 / 2}(y)\right\}^{2}+L\left\{\zeta_{\nu-1 / 1}^{\prime}(y)\right\}^{2}-\left(M_{1}+M_{2}\right) \zeta_{\nu-1 / 2}(y) \zeta_{\nu-1 / 2}^{\prime}(y)}{N \eta_{\nu-1 / 2}(y) \zeta_{\nu-1 / 2}(y)+L \zeta_{\nu-1 / 2}^{\prime}(y) \eta_{\nu-1 / 2}^{\prime}(y)-M_{1} \zeta_{\nu-1 / 2}(y) \eta_{\nu-1 / 2}^{\prime}(y)-M_{2} \zeta_{\nu-1 / 2}^{\prime}(y) \eta_{\nu-1 / 2}(y)}
$$

where

$$
y=2 \pi(R+H) / \lambda
$$

and the layer functions $L, M_{1}, M_{2}, N$ are defined by (12) in terms of functions $u(\gamma), v(\gamma)$ which satisfy equation (18) in which now

and

$$
\left.\begin{array}{l}
\alpha=\frac{1}{2} \mu_{N_{m}}\left(\Lambda^{2}-1\right), v=\gamma\left(2 \mu_{N_{m}}\right)^{1 / 2} \\
\Lambda=f\{2(\rho-\epsilon)\}^{1 / 2} / f_{N_{m}, \rho}=h / R
\end{array}\right\}
$$

In deriving (47) from equation (40) of the abovereferenced Northover paper we are taking $y_{1}=y=2 \pi$ $(R+H) / \lambda$ since the layer thickness is so small in comparison with/its height. The $\zeta, \eta$ functions are the Hankel-Nielson Bessel functions defined in the above referenced paper.

7 For example, the occasional reception of BBC television in the U.S.A. 
In the present applications, $x^{2 / 3} \rho$ is very large (e.g., if $h=80 \mathrm{~km}$ and $\lambda=7 \mathrm{~m}$, it is about 364 ) and it may then be shown (c.f., the above-referenced paper) that, for the dominant terms of (45), $X_{j}+i Y_{j}$ is small. Hence for these terms

$$
\begin{gathered}
2^{1 / 4} e^{-i \pi / 4} \zeta_{\nu-1 / 2}(y) \sim(\rho-\epsilon)^{-1 / 4} \exp \left\{-\frac{2}{3} \sqrt{2} i x(\rho-\epsilon)^{3 / 2}\right\} \\
2^{1 / 4} e^{i \pi / 4} \eta_{\nu-1 / 2}(y) \sim(\rho-\epsilon)^{-1 / 4} \exp \left\{\frac{2}{3} \sqrt{2} i x(\rho-\epsilon)^{3 / 2}\right\}
\end{gathered}
$$

and therefore (45) and (47) become,

$$
\begin{array}{r}
\frac{\zeta_{\nu-1 / 2}(x)}{\eta_{\nu-1 / 2}(x)} \doteqdot \frac{N-2(\rho-\epsilon) L+i[2(\rho-\epsilon)]^{1 / 2}\left(M_{2}+M_{1}\right)}{N+2(\rho-\epsilon) L+i\{2(\rho-\epsilon)\}^{1 / 2}\left(M_{2}-M_{1}\right)} \\
\exp \left\{-\frac{4}{3} i x \sqrt{2}(\rho-\epsilon)^{3 / 2}\right\}=-i \overline{\mathbb{H}} \\
\exp \left\{-\frac{4}{3} i \sqrt{2}(P-X+i Y)^{3 / 2}\right\}
\end{array}
$$

where $P=x^{2 / 3} \rho$, and if is the complex reflection coefficient of a ray incident upon the layer at an obliquity $\chi_{1}$ defined by

$$
\chi_{1}^{2}=2\left(\rho-\epsilon_{\jmath}\right) \doteqdot 2 \rho .
$$

Thus

$$
\Lambda=f \chi_{1} / f_{N_{m}} \doteqdot f \sqrt{2 \rho} / f_{N_{m}} .
$$

Finally, $\mathscr{F}_{j}$ is defined by (c.f. the above-referenced paper)

$$
\left.\mathscr{F}_{j}=1+\frac{1}{2} i\left\{\eta_{\nu-1 / 2}\right\}(x)\right\}^{2}\left(\frac{\partial k}{\partial s}\right)_{s=\nu}
$$

Unfortunately, the field series (45) does not admit of a straightforward evaluation as no simple formula for $\mathscr{F}_{j}$ can be found. We must therefore be content with a rather rough discussion. Since $P$ is very large and $X_{j}+i Y_{j}$ is small, it is evident from section 7 of the above-referenced paper that the curvilinear attenuation coefficient $x^{\frac{1}{3}} Y_{j}$ of the principal "modes" is approximately

$$
\frac{x^{1 / 3}}{2 \sqrt{2 P}} \log (1 /|\not|)
$$

i. e.,

$$
\frac{1}{2 \sqrt{2 \rho}} \log (1 /|R|) \text { nepers/radian }
$$

where $R$ is now the complex reflection coefficient at the obliquity given by (50).

\subsection{Discussion of the BBC Freak Transmission of 1949}

In 1949 the television transmission of the Boat Race was picked up in South Africa. The above theory may be used to provide an explanation of this (and similar infrequent phenomena) on the hypothesis that a thin ionic layer of sporadic $E$ ionization extended over most of the path (or at any rate over those parts of it near the widely separated points necessary for a multi-hop transmission over this path).

Taking $f_{N_{m}}=3 \mathrm{Mc} / \mathrm{s}$, and $h=80 \mathrm{~km}$, we find that $\Lambda=2.26$ for $\lambda=7 \mathrm{~m}$. For this value of $\Lambda$ it can be shown from (44) that $\mu_{M}=0.2182$ and $|R|_{M}=0.0443$. Thus, by (53) the smallest possible value of the curvilinear attenuation coefficient is 9.86 nepers/radian, provided that the layer thickness is then near $7 \mathrm{~m}$. Small though this attenuation rate is, it still gives a somewhat large signal loss over this long transmission path. If $f_{N_{m}}=4 \mathrm{Mc} / \mathrm{s}$, we find $\Lambda=1.7, \mu_{M}=0.4082$, and $|R|_{M}=0.1058$. The attenuation coefficient is now 7.1 nepers/radian provided that the layer thickness is now near $10 \mathrm{~m}$. Finally, in the case of the sporadic $E$ layer ionization as intense as that described by Bennington, it will not be unreasonable to take $7 \mathrm{Mc} / \mathrm{s}$ as a typical value of $f_{c_{m}}$. Here, $h=$ $115 \mathrm{~km}$ and we find that $\Lambda=1.17$ for $\lambda=7 \mathrm{~m}$. For this value of $\Lambda$ we have $\mu_{M}$ about 1.5 and $|R|_{M}=0.38$. The attenuation coefficient of the principal modes is now 2.55 nepers/radian provided that the layer thickness is about $21 \mathrm{~m}$.

\section{Critical Appreciation of the Results}

As has been already pointed out, it is impossible to discuss the field strength at distant points around the earth's curve caused by reflection from a thin elevated ionic layer with any degree of precision, because it is so difficult to estimate the values of the amplitude factors $\mathscr{F}_{j}$. But even if this were not so, it would still be impossible to obtain an elementary expression for the field strength since the attenuation coefficients of the terms at the beginning of the field series (45) decrease so slowly that there are many "modes" having nearly the same low attenuation coefficient (53). These modes will therefore be of roughly the same order of smallness, but their sum will fluctuate as the transmission distance is increased owing to variation with distance of their relative phases. We should, therefore, expect the field strength to exhibit maxima when these modes were most nearly in phase, and these maxima probably occur when the receiver is near those points on the path which can be reached by the smallest number of transmitter-receiver ray reflections.

When these principal modes reinforce one another, we may reasonably expect that their sum can be approximated to by a single term containing the attenuation factor $(\theta / 2 \sqrt{2} \rho) \log (1 /|R|)$ given by (53).

For the London-Capetown great-circle route the angular distance $\theta$ is very approximately 1.49 radians so, according to the above calculations of (53), we should expect the strength of a field strength peak at this distance to compare very unfavorably with the field strength near the limit of "ground wave" ranges when $f_{c_{m}}$ is $3 \mathrm{Mc} / \mathrm{s}$ but quite favorably when $f_{c_{m}}$ is $7 \mathrm{Mc} / \mathrm{s}$. The above argument is necessarily lacking in precision, but the conclusion reached can be seen to be problably true from ray theory considerations since we have already found that the greatest values possible for the reflection coefficients of rays which strike the layer at maximum obliquity 
(these are the ones likely to carry the furthest) are 0.0443 for $f_{N_{m}}=3 \mathrm{Mc} / \mathrm{s}$ but 0.38 for $f_{N_{m}}=7 \mathrm{Mc} / \mathrm{s}$.

It seems, therefore, that the hypothesis of the existence of suitably intense sporadic- $E$ formations over the transmission path might well account for freak propagation of the kind described above. It must be remembered, however, that the phenomenon can only be expected to occur infrequently as, not only is it necessary for the sporadic- $E$ to be suitably distributed over the transmission path, but it must also be of the right order of thickness and of exceptionally high intensity.

\section{Appendix 1}

Relation (25), which is equivalent to eq (7) of Rydbeck's paper, can be written

$$
\begin{aligned}
v(\gamma)=\frac{1}{\sqrt{2 \pi}} e^{-\frac{\alpha \pi}{2}+\frac{i \pi}{4}} \Gamma( & \left.-i \alpha+\frac{1}{2}\right) u(\gamma) \\
& +\frac{1}{\sqrt{2 \pi}} e^{\frac{\alpha \pi}{2}-\frac{i \pi}{4}} \Gamma\left(-i \alpha+\frac{1}{2}\right) u(-\gamma)
\end{aligned}
$$

or, say,

$$
(\mathrm{I})=(\mathrm{II})+(\mathrm{III}) \text {. }
$$

According to this writer, (III) represents the "transmitted" wave, (I) the "original" (upgoing) wave, and (II) the "reflected" (downgoing) wave.

The reflection coefficient $R$ is then said to be given by the absolute value of the ratio of the constant part of (II) to that of (I). This gives our formula (39), which is applicable only under certain circumstances. The present writer cannot see the validity of thus forcing a theorem of pure mathematics (which is an abstract system based on abstract axioms) to bear a direct meaning in a field of physics (an experimental science): indeed, as noted below, this kind of treatment leads to absurd results. Instead, a careful setting down of the four boundary conditions at the layer edges is required, as in our text, but boundary conditions are not even mentioned in Rydbeck's paper. Epstein (pp. 628, $629 \mathrm{ff}$. of his paper) likewise tries to interpret the ratio of the constants in a purely mathematical continuation relation obeyed by one of the functions in the layer, as a "reflection coefficient." This would perhaps be alright if the same analytic law for the layer extended to all heights but it does not-it extends only to the layers boundaries. A different differential equation (with different solutions) is applicable outside these boundaries and so there is no alternative to the proper application of the usual boundary conditions of electromagnetic theory, It is, therefore, likewise impossible for the present writer to have any confidence in Epstein's results.

Reverting to Rydbeck's paper, the present theory shows that his formula (9), page 347 , for the reflection coefficient is correct only when $\Lambda^{2}-1 \ll 1 / \mu_{N_{m}}(\Lambda>1)^{8}$ and then only when $\mu_{N m} \Lambda^{3}$ is not small. (see text comments after equation 39). His formula makes $R \rightarrow 1 / \sqrt{2}$ as the layer thickness is made to tend to zero-a conclusion that is patently absurd.

\section{Appendix 2. Note on the "Spreading Condition" Appropriate to Curved Earth Propagation}

As has already been mentioned, it is essential that the reflecting sporadic- $E$ ionization should be sufficiently well developed in the regions which surround the reflection points of the rays of maximum obliquity. The condition for this is similar to the corresponding condition for good propagation of meter wave VHF by high level tropospheric inversions [Northover, 1952] but it is more stringent because the sporadic- $E$ layer cannot be treated as a discontinuity. The analysis is rather long and will not be reproduced here owing to lack of space. The condition is, that the linear dimensions of the sporadic- $E$ ionization region near the reflection points of the rays of maximum possible obliquity should be large compared with $\left(R^{5 / 6} \lambda^{2 / 3}\right) / h^{1 / 2}$.

\section{References}

Beghian, L. E., and F. H. Northover, The reflection properties of sporadic- $E$ layers, Admiralty Signal and Radar Establishment, London, Photostat No. P. 146 (1943).

Bennington, T. W., The propagation of $\mathrm{VHF}$ via sporadic $=E$, "Wireless World," p. 5 (Jan. 1952).

Epstein, P., Reflection of plane waves in an inhomogeneous absorbing medium, Proc. Nat. Acad. Sci. U.S. 16, 627 (1930)

Hartree, D. R., The propagation of electromagnetic waves in a stratified medium, Proc. Cambridge Phil. Soc. 25, 97 (1929)

Langer, R. E., On the correction formulas and solutions of the wave equation, Phys. Rev. 51, 669 (1937).

Northover, F. H., The anomalous propagation of radio waves in the 1-10 metre band, J. Atmospheric and Terrest. Phys. \%, No. 2, 106-129 (1952).

Rydbeck, O. E. H., The reflection of electromagnetic waves from a parabolic ionised layer, Phil. Mag. 34, 342 (1943).

Whittaker, E. T. and G. N. Watson, Modern analysis, p. 347, Cambridge Univ. Press, 1927.

8 Only the second restriction applies when $0<\Lambda \leq 1$. 